UTHEP-654

\title{
Gluon scattering amplitudes from gauge/string duality and integrability
}

\author{
Yuji Satoht \\ Institute of Physics, University of Tsukuba \\ Tsukuba, Ibaraki 305-8571, Japan
}

\begin{abstract}
We discuss gluon scattering amplitudes/null-polygonal Wilson loops of $\mathcal{N}=4$ super Yang-Mills theory at strong coupling based on the gauge/string duality and its underlying integrability. We focus on the amplitudes/Wilson loops corresponding to the minimal surfaces in $A d S_{3}$, which are described by the thermodynamic Bethe ansatz equations of the homogeneous sine-Gordon model. Using conformal perturbation theory and an interesting relation between the $g$-function (boundary entropy) and the T-function, we derive analytic expansions around the limit where the Wilson loops become regular-polygonal. We also compare our analytic results with those at two loops, to find that the rescaled remainder functions are close to each other for all multi-point amplitudes.
\end{abstract}

July 2012

\footnotetext{
${ }^{\ddagger}$ ysatoh@het.ph.tsukuba.ac.jp

Contribution to the proceedings of "Progress in Quantum Field Theory and String Theory", April 3-7, 2012, Osaka City University, Osaka, Japan.
} 


\section{Introduction}

In this talk, we would like to discuss gluon scattering amplitudes of four-dimensional $\mathcal{N}=4$ super Yang-Mills theory (SYM) at strong coupling by using the gauge/string duality and its integrability. This talk is based on Refs. [1 4].

Let us start this talk by a rather general introduction to this subject. Already almost ten years ago, some integrability was discovered in the gauge/string duality or the AdS/CFT correspondence [5, 6]. This discovery of the integrability opened up new dimensions in the study of the gauge/string duality. For example, owing to the integrability, one can now discuss the gauge/string duality beyond supersymmetric sectors in a precise manner. Moreover, one can now quantitatively analyze strongcoupling dynamics of a gauge theory by using the integrability. Besides, by deeply understanding the gauge/string duality, one may expect to obtain useful insights into a lot of applications.

In fact, very impressive results about the spectrum of the planar AdS/CFT correspondence have been obtained [7] 1] Namely, one can now calculate/analyze the spectrum of single-trace operators of $\mathcal{N}=4 \mathrm{SYM}$ or of the superstrings on $A d S_{5} \times S^{5}$ for an arbitrary 't Hooft coupling in the planar limit. The spectrum is obtained by solving a set of integral equations of the type called the thermodynamic Bethe ansatz (TBA) equations [8], which typically appear in the study of finite size effects of two-dimensional integrable models. The spectrum has also been checked for simple operators on the weak-coupling side up to five loops [9, 10]. The results are really remarkable.

Given this success on the spectrum, one may well expect that the integrability should be useful also for other aspects or applications of the gauge/string duality. It has turned out that this is indeed the case, and we can now analyze gluon scattering amplitudes/null-polygonal Wilson loops of $\mathcal{N}=4 \mathrm{SYM}$ at strong coupling by using the integrability. An overview is as follows: By the AdS/CFT correspondence, the scalar part of the the maximally-helicity-violating (MHV) gluon scattering amplitude 2 at strong coupling is represented by the area of the minimal surfaces in $A d S_{5}$ which have a null-polygonal boundary on the boundary of $A d S_{5}$ [11]. These minimal surfaces also give the expectation values of the Wilson loops along the null-polygonal boundary according to the AdS/CFT correspondence, implying an equivalence between the amplitudes and the Wilson loops. The minimal surfaces are then described by a set of integral equations of the TBA type due to the integrability [1, 12, 14]. Schematically,

\footnotetext{
${ }^{1}$ See also the contributions to the proceedings by G.E. Arutyunov, V. Kazakov and R. Suzuki.

${ }^{2}$ Regarding the MHV amplitude, see also the contribution by T.R. Taylor.
} 


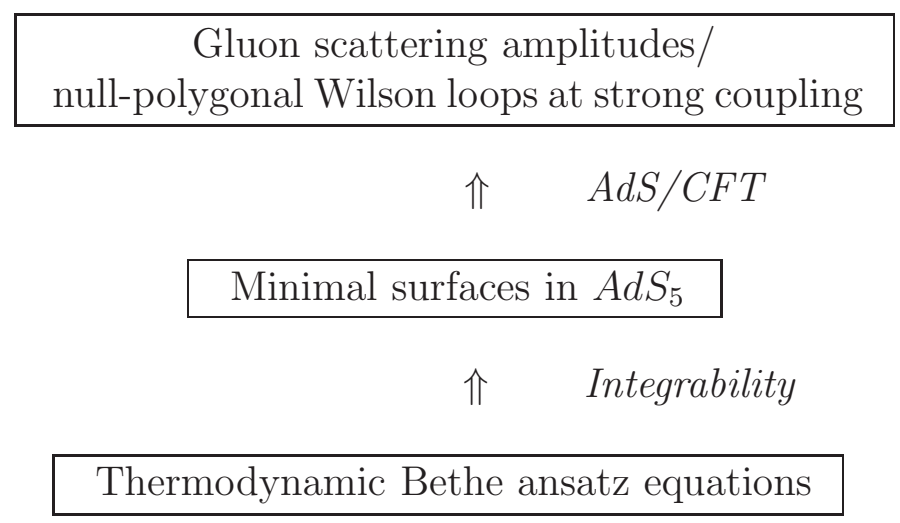

The TBA equations for the amplitudes/Wilson loops are different from those for the spectral problem. However, it is interesting to see that TBA equations appear again in a different context of the AdS/CFT correspondence.

In the following, we discuss the MHV amplitudes/null-polygonal Wilson loops of $\mathcal{N}=4 \mathrm{SYM}$ at strong coupling by using the underlying two-dimensional integrable models and conformal filed theories (CFTs). In particular, we derive analytic expansions of the amplitudes/Wilson loops around certain kinematic points corresponding to regular-polygonal Wilson loops. We focus on the case where the momenta of the external particles are contained in a two-dimensional subspace of four-dimensional Minkowski space, in other words, where the minimal surfaces are included in $A d S_{3}$ within $A d S_{5}$. We also compare our analytic results with those at two loops, and find that the rescaled remainder functions are close to each other for all multi-point amplitudes.

Before moving on to the discussion below, we would like to mention that the integrability has recently been applied also to the computation of the correlation functions and the quark anti-quark potentials (or the cusp anomalous dimensions) 3 The application of the integrability is now being expanded in this way. We would also like to mention that the development on the strong-coupling/string side is stimulating the study of $\mathcal{N}=4 \mathrm{SYM}$ at weak coupling.

The plan of the rest of this talk is as follows. In section 2, we briefly summarize the gluons scattering amplitudes/Wilson loops of $\mathcal{N}=4 \mathrm{SYM}$ at strong coupling in the context of the AdS/CFT correspondence. In section 3, we summarize how the amplitudes at strong coupling are obtained by using the integrability or the TBA equations. In section 4, which is the main part of this talk, we derive the analytic expansions of the amplitudes at strong coupling by using the two-dimensional integrable models and CFTs associated with the minimal surfaces. In section 5, we summarize the results of the lower-point cases of the eight- and ten-point amplitudes. In section

\footnotetext{
${ }^{3}$ See also the contribution by S. Komatsu.
} 


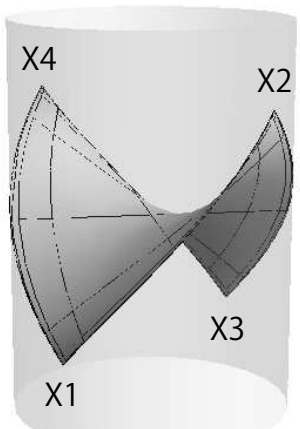

Figure 1: Null-polygonal minimal surface for four-point amplitude. The AdS space is represented by the solid cylinder, whereas the $A d S$ boundary by the side of the cylinder.

6, we compare our analytic results at strong coupling with those at two loops. We conclude with a summary and discussion in section 7 .

\section{Gluon scattering amplitudes at strong coupling}

We consider gluon scattering amplitudes of the four-dimensional maximally supersymmetric, i.e., $\mathcal{N}=4, S U\left(N_{c}\right)$ SYM in the planar limit $N_{c} \rightarrow \infty$ with the 't Hooft coupling $\lambda:=g_{Y M}^{2} N_{c}$ being fixed. For a review, see for example Ref. [15]. Since $\mathcal{N}=4$ SYM is a massless gauge theory, the amplitudes contain infrared divergences. These divergences are first to be regularized, but cancel each other in infrared-safe quantities.

In this talk, we are interested in a class of the amplitudes where all the helicity is the same except for two particles, i.e., the MHV amplitude. In the planar limit, one can factorize the tree amplitude from the MHV amplitude. By the AdS/CFT correspondence, the remaining scalar part of the amplitude at strong coupling is given by the (regularized) area, $A$, of minimal surfaces in $A d S_{5}$ [11]:

$$
\mathcal{M} \sim e^{-\frac{\sqrt{\lambda}}{2 \pi} A}
$$

In Fig. 1, we show an example of a minimal surface representing a four-point amplitude. As shown in the figure, the surface extends to the $A d S$ boundary, where the surface has a null-polygonal boundary and cusps. The sides of the surface, or the differences of the cusp points, correspond to the momenta of the external particles,

$$
x_{i+1}^{\mu}-x_{i}^{\mu}=2 \pi k_{i}^{\mu}
$$

Thus, roughly speaking, the $\tilde{n}$-point amplitudes at strong coupling are the $\tilde{n}$-cusp minimal surfaces in $A d S$. Since the minimal surfaces give expectation values of the 
Wilson loops at strong coupling along the boundary via the AdS/CFT correspondence, the above formula implies an equivalence of the MHV amplitude and the null-polygonal Wilson loops. This has been confirmed also at weak coupling [16]. Consequently, we may say that we are considering the Wilson loops of $\mathcal{N}=4 \mathrm{SYM}$ instead of the amplitudes.

In Ref. [11, four-point amplitudes were computed according to (2.11). A precise agreement was then found with the structure predicted by the Bern-Dixon-Smirnov (BDS) conjecture [17], which proposes the form of the MHV amplitude to all orders in perturbation. It turned out, however, that the BDS formula is modified for higherpoint amplitudes [18]. This deviation from the BDS formula is now called the remainder function. Because of the anomalous dual conformal Ward identities [19], this remainder function should be a function of the cross-ratios of the cusp coordinates in (2.2), which correspond to the momenta of the particles. The dual conformal symmetry also assures that the BDS formula is exact for $\tilde{n} \leq 5$. At $\tilde{n}=6$, the existence of the remainder function has indeed been confirmed [20, 21].

Once given the BDS formula, to compute the amplitudes is the same as to compute the remainder function. Thus, our task is to determine the remainder function as a function of the cross-ratios of $x_{i}^{\mu}$.

\section{Scattering amplitudes from TBA system}

After the work of Ref. [11], there were attempts at extending the strong-coupling computation to higher-point amplitudes. Though a special six-cusp minimal surface has been constructed [22, 23], it turned out that it is very difficult to construct the minimal surfaces with a null-polygonal boundary. Remarkably, it was however shown in Ref. [12] that one can calculate the area of the minimal surfaces by using integrability without knowing the explicit form of the surfaces.

In the following, we would like to explain how to carry out this program, focusing on the case where the minimal surfaces are contained in $A d S_{3} \subset A d S_{5}$ for simplicity. This corresponds to the case where the momenta of the external particles are included in a two-dimensional subspace $\mathbb{R}^{1,1}$ of four-dimensional Minkowski space. Thus, we are still considering four-dimensional physics but with special kinematics. In this case, to satisfy the momentum conservation, the number of the external particles or the cusp points becomes even: $\tilde{n}=2 n$. Here, we also introduce two light-cone coordinates $x^{ \pm}$on the boundary of $A d S_{3}$. The boundary of the minimal surfaces is then parameterized as in Fig. 2. The boundary of the surface closes at infinity in these coordinates. We note that the amplitudes depend on the momenta through the cross-ratios of $x_{i}^{ \pm}$, though. 


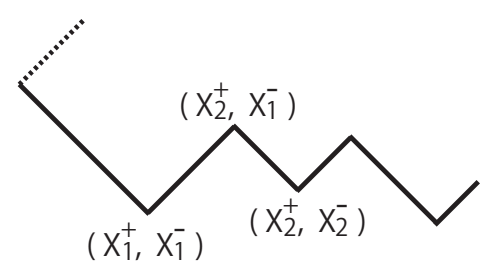

Figure 2: Boundary of null-polygonal minimal surface in $A d S_{3}$.

\subsection{TBA equations for minimal surfaces in $A d S_{3}$}

In Ref. [12], the amplitudes corresponding to the minimal surfaces in $A d S_{3}$ were analyzed. The classical string equations of motion describing the minimal surfaces were reduced to the $s u(2)$ Hitchin system or a generalized sinh-Gordon equation via the Pohlmeyer reduction. The regularized area was divided into several terms, and they were explicitly evaluated for the eight-point amplitudes with the help of the results on the moduli space of a four-dimensional $\mathcal{N}=2$ supersymmetric theory [24]. The explicit form of the surfaces is not needed in this analysis, as mentioned.

The analysis in Ref. [12] was extended to the minimal surfaces in $A d S_{5}$ in Ref. [13], where the Pohlmeyer reduction led to the su(4) Hitchin system. Interestingly, it was shown there that a non-trivial part of the regularized area for the six-point amplitudes is obtained by solving a set of integral equations, which turn out to be the TBA equations associated with the $\mathbb{Z}_{4}$-symmetric two-dimensional integrable model [25]. Following this work, the integral equations for the 10- and 12-cusp minimal surfaces in $A d S_{3}$ were derived in Ref. [1]. The general case of the $\tilde{n}$-cusp minimal surfaces in $A d S_{5}$ was studied in Ref. [14]. For the minimal surfaces in $A d S_{3}$ and $A d S_{4}$, the integral equations obtained there were identified in Ref. [1] with the TBA equations of a two-dimensional integrable model called the homogeneous-sine Gordon (HSG) model [26]. In the rest of this section, we follow the results in Ref. [14].

In order to calculate the $2 n$-point amplitudes corresponding to the minimal surfaces in $A d S_{3}$, one first needs to solve the following set of integral equations:

$$
\log \tilde{Y}_{s}(\theta)=-\left|m_{s}\right| \cosh \theta+\sum_{r=1}^{n-3} K_{s r} * \log \left(1+\tilde{Y}_{r}\right)
$$

where $s=1, \ldots, n-3, \tilde{Y}_{0}=\tilde{Y}_{n-2}=0$, and $*$ stands for the convolution, $f * g:=\int f(\theta-$ $\left.\theta^{\prime}\right) g\left(\theta^{\prime}\right) d \theta^{\prime}$. The parameter $\theta$ is an auxiliary parameter called the spectral parameter. The complex parameters $m_{s}=\left|m_{s}\right| e^{i \varphi_{s}}$ correspond to the shape of the minimal surfaces, which gives the momenta of the external particles through (2.2). By tildes, we denote the shift of the argument of the Y-functions, $Y_{s}(\theta)$, as $\tilde{Y}_{s}(\theta):=Y_{s}\left(\theta+i \varphi_{s}\right)$. The kernels 


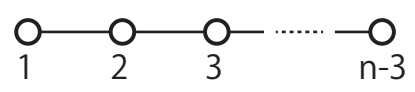

Figure 3: Dynkin diagram representing interaction among $Y_{s}$.

in the convolution are

$$
K_{s r}(\theta)=\frac{I_{s r}}{2 \pi \cosh \left(\theta+i \varphi_{s}-i \varphi_{r}\right)},
$$

where $I_{s r}=\delta_{s, r+1}+\delta_{s, r-1}$ is the incidence matrix of the $A_{n-3}$ algebra. Thus, the "interaction" among the Y-functions are represented by the Dynkin diagram of $A_{n-3}$ (see Fig. 3). Precisely, the form of (3.1) is valid for $\left|\varphi_{s}-\varphi_{s \pm 1}\right|<\pi / 2$. Outside this range, it is obtained by continuation, to pick up contributions of the poles in the kernels.

Under the condition that $\tilde{Y}_{s}(\theta)$ are analytic for $|\operatorname{Im} \theta|<\pi / 2$, the integral equations are converted into a set of algebraic equations called the $\mathrm{Y}$-system [27]:

$$
Y_{s}^{[+1]}(\theta) Y_{s}^{[-1]}(\theta)=\left(1+Y_{s+1}(\theta)\right)\left(1+Y_{s-1}(\theta)\right),
$$

where the bracket stands for the shift of the argument,

$$
f^{[k]}(\theta):=f\left(\theta+\frac{\pi i}{2} k\right) .
$$

The Y-functions represent the cross-ratios of the cusp coordinates extended by the spectral parameter. Indeed, one finds that

$$
\begin{aligned}
Y_{2 r+1}^{[-1]}(0) & =\frac{x_{r,-r-1}^{+} x_{r+1,-r-2}^{+}}{x_{r, r+1}^{+} x_{-r-2,-r-1}^{+}}, & Y_{2 r+1}^{[0]}(0) & =\frac{x_{r,-r-1}^{-} x_{r+1,-r-2}^{-}}{x_{r, r+1}^{-} x_{-r-2,-r-1}^{-}}, \\
Y_{2 r}^{[0]}(0) & =\frac{x_{r,-r}^{+} x_{r+1,-r-1}^{+}}{x_{r, r+1}^{+} x_{-r-1,-r}^{+}}, & Y_{2 r}^{[1]}(0) & =\frac{x_{r,-r}^{-} x_{r+1,-r-1}^{-}}{x_{r, r+1}^{-} x_{-r-1,-r}^{-}},
\end{aligned}
$$

where $x_{i, j}^{\mu}:=x_{i}^{\mu}-x_{j}^{\mu}$, and the indices for the cusps are labeled modulo $n$. For example, $Y_{1}(-\pi i / 2)=x_{15}^{+} x_{67}^{+} / x_{56}^{+} x_{17}^{+}, Y_{1}(0)=x_{15}^{-} x_{67}^{-} / x_{56}^{-} x_{17}^{-}$for $n=7$. Graphically, these crossratios are represented by the tetragons inside the $n$-gons formed by $x_{i}^{ \pm}$. In Fig. 4 , we show an example of $n=7$.

As mentioned above, the equations (3.1) are nothing but the TBA equations of the HSG model. In the context of the two-dimensional integrable model, $m_{s}$ are the (complexified) mass parameters, $\theta$ is the rapidity, and $Y_{s}$ are the exponentials of the pseudo-energies.

\subsection{Remainder function}

Once the Y-functions are obtained by solving the TBA equations (3.1), one can write down the formula of the remainder function, which is defined by the difference between 


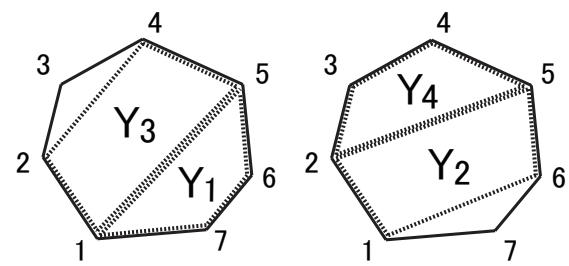

Figure 4: Graphical representation of Y-functions for $n=7$. The $i$-th vertex stands for $x_{i}^{+}$or $x_{i}^{-}$. At special values of $\theta, Y_{s}$ give the cross-ratios formed by the cusp coordinates at the corners of the corresponding tetragons. We omit the shifts $[k]$ of $Y_{s}^{[k]}$.

the amplitude and the BDS formula. For the time being, we focus on the case of the $2 n$-point amplitudes with $n$ odd. In this case, the formula reads

$$
\begin{aligned}
R_{2 n} & :=A \text { (amplitude })-A_{\mathrm{BDS}}(\mathrm{BDS} \text { formula }) \\
& =\frac{7 \pi}{12}(n-2)+A_{\text {periods }}+\Delta A_{\mathrm{BDS}}+A_{\text {free }} .
\end{aligned}
$$

The overall coupling constant $\sqrt{\lambda}$ has been omitted above. The first term is a constant. The second term, which is given by

$$
A_{\text {periods }}=-\frac{1}{4} m_{r} I_{r s}^{-1} \bar{m}_{s}
$$

comes from period integrals over an auxiliary hyperelliptic curve $y^{2}=p(z)$ with $p(z)$ being a polynomial of degree $n-2$. The third term, which is given by

$$
\Delta A_{\mathrm{BDS}}=\frac{1}{4} \sum_{i, j=1}^{n} \log \frac{c_{i, j}^{+}}{c_{i, j+1}^{+}} \log \frac{c_{i-1, j}^{-}}{c_{i, j}^{-}}
$$

comes from the difference between a term satisfying the anomalous dual conformal Ward identities and a similar term in the BDS formula. Here, $c_{i, j}^{ \pm}$are the sequential cross-ratios formed by the nearest neighbor distances of $x_{i}^{ \pm}$,

$$
c_{i, j}^{ \pm}:=\frac{x_{i+2, i+1}^{ \pm} x_{i+4, i+3}^{ \pm} \cdots x_{j, i}^{ \pm}}{x_{i+1, i}^{ \pm} x_{i+3, i+2}^{ \pm} \cdots x_{j, j-1}^{ \pm}} .
$$

Similarly to $Y_{s}$ in Fig. 4, these are graphically represented by polygons inside the $n$-gons formed by $x_{i}^{ \pm}$. In Fig. 5 , we show an example of $c_{1,6}^{ \pm}$for $n=7$. The last term is given by the following integral,

$$
A_{\text {free }}=\sum_{s=1}^{n-3} \int_{-\infty}^{\infty} \frac{d \theta}{2 \pi}\left|m_{s}\right| \cosh \theta \log \left(1+\tilde{Y}_{s}(\theta)\right) .
$$

Interestingly, this is nothing but the free energy associated with the TBA system (up to a factor). From these expressions, we find that there are two non-trivial parts, $\Delta A_{\mathrm{BDS}}$ and $A_{\text {free }}$, in expressing the remainder function in terms of the mass parameters $m_{s}$. 


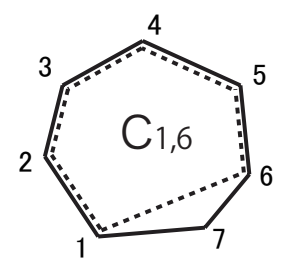

Figure 5: Graphical representation of cross-ratios $c_{1,6}^{ \pm}$for $n=7$. We omit superscripts \pm .

Given the formula of the remainder function, what we should do is to solve the TBA equations. They are solved numerically by iterations, to get numbers of $R_{2 n}$ for given $m_{s}$. The TBA equations (3.1) are also solved exactly in the limit where all $m_{s}$ approach 0 or $\infty$. The former limit corresponds to the UV limit where the twodimensional integrable model reduces to a CFT, or to the limit where the corresponding Wilson loops become regular-polygonal. The latter corresponds to the IR limit where the particles in the two-dimensional integrable model become free massive particles, or to soft limits of the SYM theory.

However, the solutions to the TBA system (3.1) have not been fully understood yet. To find the momentum dependence of the remainder function and its structures, one also needs to find the relation between $m_{s}$ and the momenta, since the TBA equations are solved for given $m_{s}$. Here, one may need some analytic data. In addition, interesting analytic results have been obtained at two loops [28 31]. Taking these into account, it would be worthwhile to explore analytic results at strong coupling besides in the special limits $m_{s} \rightarrow 0, \infty$.

This is the subject of the rest of this talk. In particular, we discuss analytic expansions of the remainder function around the UV/CFT limit $m_{s} \rightarrow 0$. For later use, here we introduce the mass scale $M$, the length scale $L$ which corresponds to the inverse temperature of the TBA system, the dimensionful mass parameters $M_{s}$ and the relative masses $\tilde{M}_{s}$, so that

$$
m_{s}=M_{s} L=\tilde{M}_{s} M L
$$

In terms of these parameters, the CFT limit we consider is represented as

$$
l:=M L \rightarrow 0 \text {. }
$$

\section{Analytic expansions of amplitudes}

Now, let us move on to the discussion on the analytic expansion [3, 4]. A basis of the expansion is the fact that the integral equations for the minimal surfaces in $A d S_{3}$ are 
the TBA equations of the HSG model associated with the coset $\widehat{s u}(n-2)_{2} /[\widehat{u}(1)]^{n-3}[1]$ . Schematically,

\begin{tabular}{|c|c|}
\hline TBA for $A d S_{3}$ \\
2n-pt. amplitudes
\end{tabular}$\Longleftarrow \quad$ HSG from $\frac{\widehat{s u}(n-2)_{2}}{[\widehat{u}(1)]^{n-3}}$

Precisely, in the TBA equations for the minimal surfaces, the resonance parameters are purely imaginary. Though physical interpretation of the imaginary resonance parameters is not clear at present, they are irrelevant to the following discussion: they are first set to be zero and, after the expansion is obtained, they are recovered so as to maintain symmetries. Vanishing imaginary resonance parameters correspond to real mass parameters. We thus consider real $m_{s}$ for the time being.

Similarly, the integral equations for the $\tilde{n}$-cusp minimal surfaces in $A d S_{4}$ are the TBA equations of the HSG model associated with the coset $\widehat{s u}(\tilde{n}-4)_{4} /[\widehat{u}(1)]^{\tilde{n}-5}$ [1]. For the $A d S_{5}$ case, the corresponding integrable model for the six-cusp minimal surfaces is the $\mathbb{Z}_{4}$-integrable model with a twist [2,13], which is equivalent to the HSG model associated with $\widehat{s u}(2)_{4} /[\widehat{u}(1)]$ or corresponding complex sine-Gordon model with a twist. However, the integrable model for the general $A d S_{5}$ case has not been identified.

The HSG model associated with the coset $\widehat{s u}(n-2)_{2} /[\widehat{u}(1)]^{n-3}$ for the $A d S_{3}$ minimal surfaces is obtained by an integrable deformation of the corresponding coset $\mathrm{CFT}$ /gauged WZNW model. The action is then given by

$$
S_{\mathrm{HSG}}=S_{\mathrm{gWZNW}}+\beta \int d^{2} x \Phi
$$

where the first term on the right-hand side is the action of the gauged WZNW model and $\Phi\left(\tilde{M}_{s}\right)$ is a linear combination of the weight 0 adjoint operators, which has the coefficients depending on $\tilde{M}_{s}$ and conformal weights

$$
\Delta=\bar{\Delta}=(n-2) / n
$$

On dimensional grounds, the coupling $\beta$ takes the form

$$
\beta=-\kappa_{n} M^{2(1-\Delta)}
$$

where $\kappa_{n}$ is the dimensionless coupling.

Once we find the relation among the integral equations for the minimal surfaces, the two-dimensional integrable model and the corresponding CFT in the CFT/UV limit, we can expand the remainder function around the CFT limit by conformal perturbation theory (CPT). Here, we recall that there are two non-trivial parts in expanding $R_{2 n}$, i.e., $A_{\text {free }}$ and $\Delta A_{\mathrm{BDS}}$. Let us discuss their expansions one by one. 


\subsection{Expansion of free energy part}

Since $A_{\text {free }}$ is the free energy of the HSG model, it is straightforward to expand it around the CFT limit by using the action (4.1) and CPT. By the standard procedure [8], one has

$$
A_{\text {free }}=\frac{\pi}{6} c_{n}+f_{n}^{\text {bulk }}+\sum_{p=2}^{\infty} f_{n}^{(p)} l^{4 p / n} .
$$

The first term $c_{n}=(n-2)(n-3) / n$ is the central charge of the coset CFT for $\widehat{s u}(n-$ $2)_{2} /[\widehat{u}(1)]^{n-3}$. The second term $f_{n}^{\text {bulk }}$ is the bulk term given for $n$ odd by

$$
f_{n}^{\text {bulk }}=\frac{1}{4} m_{r} I_{r s}^{-1} \bar{m}_{s},
$$

which cancels with $A_{\text {periods }}$ in (3.7). The terms in the summation come from the perturbation by $\Phi$,

$$
f_{n}^{(p)}=\frac{\kappa_{n}^{p}}{p !}(2 \pi)^{2+2(\Delta-1) p} \int\left\langle\Phi\left(x_{1}\right) \cdots \Phi\left(x_{p}\right)\right\rangle_{\mathrm{c}} \prod_{i=2}^{p}\left|x_{i}\right|^{-2(\Delta-1)} d^{2} x_{i},
$$

where the correlators are connected ones evaluated at the CFT point and we have set $x_{1}=1$. At the lowest order, we have

$$
f_{n}^{(2)}=\frac{\pi}{6} C_{n}^{(2)} \kappa_{n}^{2} G^{2}\left(\tilde{M}_{s}\right)
$$

where

$$
C_{n}^{(2)}=3(2 \pi)^{\frac{2(n-4)}{n}} \gamma^{2}\left(\frac{n-2}{n}\right) \gamma\left(\frac{4-n}{n}\right),
$$

$\gamma(x):=\Gamma(x) / \Gamma(1-x)$, and $G\left(\tilde{M}_{s}\right)$ is a normalization factor defined through

$$
\langle\Phi(x) \Phi(0)\rangle=\frac{G^{2}\left(\tilde{M}_{s}\right)}{|x|^{4 \Delta}} .
$$

To determine the dependence on $\tilde{M}_{s}$, one needs to find the precise form of $\Phi\left(\tilde{M}_{s}\right)$ in terms of the weight 0 adjoint operators. We will come back to this issue later.

\section{2 $\Delta A_{\mathrm{BDS}}$ and T-functions}

Next, let us consider the expansion of $\Delta A_{\mathrm{BDS}}$, which is given by the sequential cross-

ratios $c_{i, j}^{ \pm}$. Interestingly, with the help of graphical representations as in Figs. 4 and 5 , one can show that these cross-ratios are directly expressed by the T-functions [32], which are related to the Y-functions as

$$
Y_{s}(\theta)=T_{s+1}(\theta) T_{s-1}(\theta), \quad 1+Y_{s}(\theta)=T_{s}^{[+1]}(\theta) T_{s}^{[-1]}(\theta) .
$$


One then finds for $n$ odd that

$$
c_{i, j}^{+}=T_{|i-j|-1}^{[i+j]}(0), \quad c_{i, j}^{-}=T_{|i-j|-1}^{[i+j+1]}(0) .
$$

Consequently, $\Delta A_{\mathrm{BDS}}$ is represented by the T-functions as

$$
\Delta A_{\mathrm{BDS}}=\frac{1}{4} \sum_{i, j=1}^{n} \log \frac{T_{|i-j|-1}^{[i+j]}}{T_{|i-j-1|-1}^{[i+j+1]}} \log \frac{T_{|i-j-1|-1}^{[i+j]}}{T_{|i-j|-1}^{[i+j+1]}},
$$

where $T_{s}^{[k]}(\theta)$ are evaluated at $\theta=0$. We see that each term in the remainder function (3.6) nicely fits into the language of two-dimensional integrable models: $A_{\text {free }}$ is the free energy, and $A_{\text {periods }}$ and $\Delta A_{\mathrm{BDS}}$ are given by the mass parameters and the T-functions, respectively. Now, we have only to expand $T_{s}$ around the CFT limit.

\subsection{Expansion of T-functions}

To expand the T-functions, we use an interesting relation [33 35] between the Tfunction and the boundary entropy or the $g$-function [36]. Since the $g$-function is regarded as a boundary contribution to the free energy, one can compute it around the CFT limit by using CPT with boundary [34,35].

The precise relation between the $g$ - and T-functions is obtained by (i) constructing the reflection factors of the HSG model which satisfy the unitarity, the crossing symmetry, and the boundary Yang-Baxter equation, (ii) deriving the integral equations for the $g$-functions associated with the boundaries corresponding to the reflection factors, and (iii) comparing those equations with those for the T-functions. Here, to satisfy the boundary Yang-Baxter equation, the imaginary resonance parameters or the phases of the mass parameters need to be vanishing, as they are in our discussion so far. For details, we refer to Ref. [3].

In expanding $T_{s}$, we then use their quasi-periodicity for $n$ odd,

$$
T_{s}^{[n]}(\theta)=T_{n-2-s}(\theta)
$$

This follows from the relations (4.10) which are written in the form of the T-system,

$$
1+T_{s+1}(\theta) T_{s-1}(\theta)=T_{s}^{[+1]}(\theta) T_{s}^{[-1]}(\theta)
$$

with $T_{0}=T_{n-2}=1$. Combining this quasi-periodicity and the structure of the CPT, the T-functions are expanded for real $m_{s}$ and $n$ odd as

$$
T_{s}(\theta)=\sum_{p, q=0} t_{s}^{(p, 2 q)} l^{(1-\Delta)(p+q)} \cosh \left(\frac{2 p}{n} \theta\right),
$$


with $t_{n-2-s}^{(p, 2 q)}=(-1)^{p} t_{s}^{(p, 2 q)}$. The T-system then fixes the lower coefficients as

$$
t_{s}^{(0,0)}=\sin \left(\frac{s+1}{n} \pi\right) / \sin \left(\frac{\pi}{n}\right)
$$

and $t_{s}^{(1,0)}=t_{s}^{(0,2)}=t_{s}^{(1,2)}=0$. Further using the results on the conformal perturbation of the $g$-function, and translating them into the expansion of the T-functions, we obtain

$$
\frac{t_{s}^{(2,0)}}{t_{s}^{(0,0)}}=-\frac{\kappa_{n} G \cdot B(1-2 \Delta, \Delta)}{2(2 \pi)^{1-2 \Delta}}\left(\frac{\sin \left(\frac{3(s+1) \pi}{n}\right)}{\sin \left(\frac{(s+1) \pi}{n}\right)} \sqrt{\frac{\sin \left(\frac{\pi}{n}\right)}{\sin \left(\frac{3 \pi}{n}\right)}}-\sqrt{\frac{\sin \left(\frac{3 \pi}{n}\right)}{\sin \left(\frac{\pi}{n}\right)}}\right),
$$

where $B(a, b)=\Gamma(a) \Gamma(b) / \Gamma(a+b)$. We note that the ratios of the sine functions come from the modular S-matrix of the coset CFT. In relation to the $g$-function, the form of $t_{s}^{(0,0)}$ in (4.16) is also interpreted in this way. From the T-system, one can also show that $t_{s}^{(0,4)}$ are given by $t_{s}^{(2,0)}$ via

$$
2 t_{s}^{(0,0)} t_{s}^{(0,4)}+\frac{1}{2}\left(t_{s}^{(2,0)}\right)^{2} \cos \left(\frac{4 \pi}{n}\right)=t_{s-1}^{(0,0)} t_{s+1}^{(0,4)}+t_{s+1}^{(0,0)} t_{s-1}^{(0,4)}+\frac{1}{2} t_{s-1}^{(2,0)} t_{s+1}^{(2,0)} .
$$

Together with (4.17), we see that all $t_{s}^{(2,0)}$ and $t_{s}^{(0,4)}$ are expressed, e.g., by $t_{1}^{(2,0)} \propto$ $\kappa_{n} G\left(\tilde{M}_{s}\right)$.

\subsection{Expansion of remainder function}

From the expansion of $A_{\text {free }}$ and the one of $\Delta A_{\mathrm{BDS}}$ via those of $T_{s}$, the remainder function is expanded around the CFT limit. After some algebras, we find that

$$
R_{2 n}=R_{2 n}^{(0)}+l^{\frac{8}{n}} R_{2 n}^{(4)}+\mathcal{O}\left(l^{\frac{12}{n}}\right)
$$

for $n$ odd and real $m_{s}$, where

$$
\begin{aligned}
& R_{2 n}^{(0)}=\frac{\pi}{4 n}(n-2)(3 n-2)-\frac{n}{2} \sum_{s=1}^{(n-3) / 2} \log ^{2}\left(\frac{\sin \left(\frac{(s+1) \pi}{n}\right)}{\sin \left(\frac{s \pi}{n}\right)}\right) \\
& R_{2 n}^{(4)}=\frac{\pi}{6} C_{n}^{(2)} \kappa_{n}^{2} G^{2}\left(\tilde{M}_{j}\right)-\frac{n}{4}\left[\sum_{s=1}^{(n-3) / 2} A_{n, s}-2\left(\frac{t_{(n-3) / 2}^{(2,0)}}{t_{(n-3) / 2}^{(0,0)}}\right)^{2} \sin ^{2}\left(\frac{\pi}{n}\right)\right]
\end{aligned}
$$

and

$$
\begin{aligned}
A_{n, s}= & {\left[\left(\frac{t_{s-1}^{(2,0)}}{t_{s-1}^{(0,0)}}\right)^{2}+\left(\frac{t_{s}^{(2,0)}}{t_{s}^{(0,0)}}\right)^{2}\right] \cos \left(\frac{2 \pi}{n}\right)-\frac{2 t_{s-1}^{(2,0)} t_{s}^{(2,0)}}{t_{s-1}^{(0,0)} t_{s}^{(0,0)}} } \\
& +\left[\left(\frac{t_{s-1}^{(2,0)}}{t_{s-1}^{(0,0)}}\right)^{2}-\left(\frac{t_{s}^{(2,0)}}{t_{s}^{(0,0)}}\right)^{2}-4\left(\frac{t_{s-1}^{(0,4)}}{t_{s-1}^{(0,0)}}-\frac{t_{s}^{(0,4)}}{t_{s}^{(0,0)}}\right)\right] \log \left(\frac{t_{s}^{(0,0)}}{t_{s-1}^{(0,0)}}\right) .
\end{aligned}
$$

The first term in the expansion $R_{2 n}^{(0)}$ gives the remainder function in the CFT limit, or for the regular-polygonal Wilson loops. We also note that $t_{s}^{(3,0)}, t_{s}^{(2,2)}$ and $t_{s}^{(4,0)}$ do not appear, and hence $R_{2 n}^{(4)}$ is expressed by $t_{1}^{(2,0)}$ or $\kappa_{n} G$. 


\section{5 $\mathbb{Z}_{2 n}$-symmetry and remainder function for complex $\boldsymbol{m}_{s}$}

The absence of $t_{s}^{(3,0)}, t_{s}^{(2,2)}, t_{s}^{(4,0)}$ in the above expansion is understood as a consequence of the $\mathbb{Z}_{2 n}$-symmetry, which is the symmetry under the cyclic shift of the cusp points: $k$-th cusp $\rightarrow(k+1)$-th cusp or, in terms of the light-cone coordinates,

$$
x_{j}^{-} \rightarrow x_{j+1}^{+}, \quad x_{j}^{+} \rightarrow x_{j}^{-} .
$$

This is concisely expressed in terms of the Y-functions as [37]

$$
Y_{s}(\theta) \rightarrow Y_{s}^{[+1]}(\theta)
$$

or in terms of the mass parameters as $m_{s} \rightarrow m_{s} / i$. Acting with this symmetry twice induces a simple translation $x_{j}^{ \pm} \rightarrow x_{j+1}^{ \pm}$. For general complex $m_{s}$, the expansion of $T_{s}$ in (4.15) is modified so that $t_{s}^{(p, 2 q)} \cosh (2 p \theta / n) \rightarrow \frac{1}{2}\left(t_{s}^{(p, 2 q)} e^{2 p \theta / n}+\bar{t}_{s}^{(p, 2 q)} e^{-2 p \theta / n}\right)$. The shift $\theta \rightarrow \theta+\pi i / 2$ in (4.23) is thus translated into the phase shift of the coefficients $t_{s}^{(p, 2 q)}, \bar{t}_{s}^{(p, 2 q)}$. Then, the non-constant invariant combinations under the $\mathbb{Z}_{2 n}$-symmetry

are only $t_{s}^{(2,0)} \bar{t}_{s}^{(2,0)}$ and $t_{s}^{(0,4)}$ up to $\mathcal{O}\left(l^{\frac{8}{n}}\right)$, which explains the terms in the expansion of $R_{2 n}$.

So far, we have considered real $m_{s}$ corresponding to the vanishing imaginary resonance parameters. The expansion for complex $m_{s}$ is also obtained so as to maintain this $\mathbb{Z}_{2 n}$-symmetry: we rewrite the expansion in terms of the $\mathbb{Z}_{2 n}$-invariant combinations of $t_{s}^{(p, 2 q)}$, and then continue the real $\tilde{M}_{s}$ in $t_{s}^{(p, 2 q)}$ to complex $\tilde{M}_{s}$. Up to $\mathcal{O}\left(l^{\frac{8}{n}}\right)$, this procedure reduces to replacing $\kappa_{n}^{2} G^{2}$ for real $m_{s}$ by $\kappa_{n}^{2} G \bar{G}$ for complex $m_{s}$. The $\mathbb{Z}_{2 n}$-symmetry strongly constrains the structure of the remainder function in this way.

\subsection{Case of $n$ even}

So far, we have focused on the case of $n$ of $2 n$ odd. For $n$ even, there are several changes. For example, the remainder function has an extra term $A_{\text {extra }}$ in addition to those in (3.6) due to a non-trivial monodromy of an auxiliary variable defined by $d w=\sqrt{p(z)} d z$. The expressions of $A_{\text {periods }}$ and $\Delta A_{\mathrm{BDS}}$ are also changed. Since $T_{n-2} \neq 1$ generally for $n$ even, the quasi-periodicity of the T-functions is also modified. Due to this, their expansions have extra factors compared with (4.15). The remaining part, which we denote by $\hat{T}_{s}$, however, has the same quasi-periodicity and the form of the expansion as in (4.13) and (4.15), respectively. We refer to Ref. [4] for derails. In any case, since the extra term $A_{\text {extra }}$ and the extra factors in $T_{s}$ are irrelevant up to $o(l)$, the expansion of $R_{2 n}$ up to $\mathcal{O}\left(l^{\frac{8}{n}}\right)$ for $n \geq 10$ is similar to the one for $n$ odd. We then obtain the 
expansion of the form (4.19) with

$$
\begin{aligned}
& R_{2 n}^{(0)}=\frac{\pi}{4 n}(n-2)(3 n-2)-\frac{n}{2} \sum_{s=1}^{n / 2-1} \log ^{2}\left(\frac{\sin \left(\frac{(s+1) \pi}{n}\right)}{\sin \left(\frac{s \pi}{n}\right)}\right) \\
& R_{2 n}^{(4)}=\frac{\pi}{6} C_{n}^{(2)} \kappa_{n}^{2} G^{2}\left(\tilde{M}_{j}\right)-\frac{n}{4} \sum_{s=1}^{n / 2-1} \hat{A}_{n, s}
\end{aligned}
$$

for $n \geq 10$. Here, $\hat{A}_{n, s}$ are give by (4.21) with $t_{s}^{(p, 2 q)}$ replaced by $\hat{t}_{s}^{(p, 2 q)}$, which are the expansion coefficients of $\hat{T}_{s}$. In the expansion up to this order, the relevant $\hat{t}_{s}^{(p, 2 q)}$ are however the same as $t_{s}^{(p, 2 q)}$ and given by (4.16), (4.17) and (4.18).

For $n=6,8$, we have also checked that various modifications in each term in $R_{2 n}$ eventually cancel each other, and we are left with the same expression as for $n \geq 10$. The relevant coefficients $\hat{t}_{s}^{(p, 2 q)}$ are the same as $t_{s}^{(p, 2 q)}$ and given by (4.16), (4.17) and (4.18) again. The case of $n=4$ is special in that the TBA system becomes trivial. In this case, the integral expression of the remainder function is given in Ref. [12], and is expanded in $l^{2}$ to all orders in Ref. [3].

The expansion for general complex $m_{s}$ is obtained by complexifying $m_{s}$ similarly to the case of $n$ odd.

\subsection{Momentum dependence}

The expansion so far is given in terms of $\left|t_{s}^{(2,0)}\right|^{2} \propto \kappa_{n}^{2} G \bar{G}$. To express the remainder function as a function of the momenta, we need to find the relation between those expansion parameters and the cross-ratios. For this purpose, we first note that the Y-functions have the quasi-periodicity,

$$
Y_{s}^{[n]}(\theta)=Y_{n-2-s}(\theta)
$$

for $n$ both odd and even. This follows from the Y-system (3.3). Then, similarly to $T_{s}$, the Y-functions for general complex $m_{s}$ are expanded around the CFT limit as

$$
Y_{s}(\theta)=y_{s}^{(0,0)}+\frac{1}{2}\left(y_{s}^{(2,0)} e^{\frac{4}{n} \theta}+\bar{y}_{s}^{(2,0)} e^{-\frac{4}{n} \theta}\right) l^{\frac{4}{n}}+\mathcal{O}\left(l^{\frac{6}{n}}\right)
$$

where $y_{s}^{(0,0)}$ are the solution to the constant Y-system,

$$
y_{s}^{(0,0)}=\sin \left(\frac{s \pi}{n}\right) \sin \left(\frac{(s+2) \pi}{n}\right) / \sin ^{2}\left(\frac{\pi}{n}\right) .
$$

From the relations (4.10), we then find that

$$
Y_{s}^{[k]}(0)=y_{s}^{(0,0)}+\cos \left(\frac{2 \pi}{n}\right) t_{s}^{(0,0)}\left(t_{s}^{(2,0)} e^{\frac{2 \pi}{n} k i}+\bar{t}_{s}^{(2,0)} e^{-\frac{2 \pi}{n} k i}\right) l^{\frac{4}{n}}+\mathcal{O}\left(l^{\frac{6}{n}}\right) .
$$


Inverting this gives

$$
\begin{aligned}
\left|t_{s}^{(2,0)}\right| l^{\frac{4}{n}} & =\frac{\delta Y_{s}^{[0]}}{2 t_{s}^{(0,0)} \cos \left(\frac{2 \pi}{n}\right) \cos \phi_{s}}, \\
\frac{2 \pi}{n} \phi_{s} & =\arctan \left(\cot \left(\frac{2 \pi}{n}\right) \frac{\delta Y_{s}^{[-1]}-\delta Y_{s}^{[1]}}{\delta Y_{s}^{[-1]}+\delta Y_{s}^{[1]}}\right),
\end{aligned}
$$

up to the present order, where we have set $t_{s}^{(2,0)}=\left|t_{s}^{(2,0)}\right| e^{i \phi_{s}}$, and $\delta Y_{s}^{[k]}$ are the deviations of the cross-ratios from the CFT/regular-polygonal limit, $\delta Y_{s}^{[k]}:=Y_{s}^{[k]}-y_{s}^{(0,0)}$. By using the relations (3.5), these are indeed expressed in terms of the cross-ratios (which depend on each other at this order through (4.28)). The momentum dependence of the remainder function is found by substituting (4.29) into the expansion.

\subsection{Mass-coupling relations}

At higher orders, the expansion may not be expressed only by $\kappa_{n} G$. Thus, we need to find the precise form of the perturbing term $\kappa_{n} \Phi$ for given $m_{s}$, i.e., the mass-coupling relation in the HSG model. This is also necessary to make contact with numerics using the TBA system (3.1) for given masses, as well as to find the connection between $m_{s}$ and the cross-ratios/shape of the minimal surfaces.

This is achieved for some cases where the TBA system has only one mass scale. A classification of such cases is given in Ref. [38. In this subsection, we set $m_{s}$ to be real again. To see this, we first parameterize $\Phi$ as

$$
\Phi=\sum_{l, \bar{l}=1}^{n-3}(\boldsymbol{\lambda})^{l}(\boldsymbol{\lambda})^{\bar{l}} \phi_{l, \bar{l}}
$$

where $\phi_{l, l^{\prime}}$ are the weight 0 adjoint operators normalized so that $\left\langle\phi_{l, \bar{l}}(z) \phi_{l^{\prime}, \bar{l}^{\prime}}(0)\right\rangle=$ $\delta_{l, l^{\prime}} \delta_{\bar{l}, \bar{l}^{\prime}}|z|^{-4 \Delta}$, and $\boldsymbol{\lambda}=\sum_{j=1}^{n-3} \tilde{M}_{j}^{1-\Delta} \hat{\boldsymbol{\lambda}}_{j}$. Classically, $\hat{\boldsymbol{\lambda}}_{j}$ are the fundamental weights of $s u(n-2)$ [39,40]. In this parameterization, one has

$$
G\left(\tilde{M}_{j}\right)=\sum_{i, j=1}^{n-1} \tilde{M}_{i}^{\frac{2}{n}} F_{i j} \tilde{M}_{j}^{\frac{2}{n}}, \quad F_{i j}:=\hat{\boldsymbol{\lambda}}_{i} \cdot \hat{\boldsymbol{\lambda}}_{j} .
$$

When the mass parameters are set to be $M_{1}=M, M_{2}=\cdots=M_{n-3}=0$, the TBA system reduces to the one for the (RSOS) ${ }_{n-2}$ scattering theory [41,42], which is regarded as a massive integrable deformation of the unitary minimal model $\mathcal{M}_{n-1, n}$ by the $\phi_{1,3}$ operator. From the result in Ref. [43, we then find that

$$
\kappa_{n} F_{11}=\frac{1}{\pi} \frac{n^{2}}{(n-2)(2 n-3)}\left[\gamma\left(\frac{3(n-1)}{n}\right) \gamma\left(\frac{n-1}{n}\right)\right]^{\frac{1}{2}}\left[\frac{\sqrt{\pi} \Gamma\left(\frac{n}{2}\right)}{2 \Gamma\left(\frac{n-1}{2}\right)}\right]^{\frac{4}{n}} .
$$


When only the $k$-th mass parameter is non-vanishing, i.e., $M_{j}=\delta_{j k} M$, the TBA system reduces to the one for an integrable deformation of the coset CFT associated with $\widehat{s u}(2)_{k} \oplus \widehat{s u}(2)_{n-2-k} / \widehat{s u}(2)_{n-2}$ by the $\phi_{1,1, \text { adj }}$ operator [44]. Using the result in Ref. [45] together with (4.32), we also find that

$$
\frac{F_{k k}}{F_{11}}=\frac{k(n-k-2)}{n-3}\left[\frac{\sqrt{\pi}}{2} \frac{\Gamma\left(\frac{n-1}{2}\right)}{\Gamma\left(\frac{k}{2}+1\right) \Gamma\left(\frac{n-k}{2}\right)}\right]^{\frac{4}{n}} .
$$

Furthermore, when $M_{1}=M_{n-3}=M, M_{2}=\cdots=M_{n-4}=0$ for $n$ odd, the TBA system reduces to the one for the magnonic $T_{(n-3) / 2}=A_{n-3} / \mathbb{Z}_{2}$ system, which is described by an integrable perturbation of the non-unitary coset CFT associated with $\widehat{s u}(2)_{n / 2-3} \oplus \widehat{s u}(2)_{1} / \widehat{s u}(2)_{n / 2-2}$ by the $\phi_{1,1, \text { adj }}$ operator, or the non-unitary minimal model $\mathcal{M}_{n-2, n}$ perturbed by the $\phi_{1,3}$ operator [38]. Then, using the result again in Ref. [45], one can derive

$$
1+\frac{F_{1, n-3}}{F_{11}}=\frac{n-2}{n-3}\left[\frac{\Gamma\left(\frac{n}{4}\right) \Gamma\left(\frac{n-1}{2}\right)}{\Gamma\left(\frac{n}{4}-\frac{1}{2}\right) \Gamma\left(\frac{n}{2}\right)}\right]^{\frac{4}{n}} .
$$

From (4.32), (4.33) and (4.34), the form of $\kappa_{n} \Phi$ is fixed in the corresponding cases. By changing the mass scale $M$, one can trace the the remainder function along the trajectories in the momentum space parameterized by the mass parameters. As we will discuss shortly, these data completely fix the mass-coupling relation for the 10-point amplitudes.

\section{Lower-point cases}

So far, we have discussed the expansion of the remainder function for general $n$ of $2 n$. In this section, we specialize to the case of $2 n=8$ and 10 [3].

\subsection{Eight-point amplitudes}

In the case of the eight-point amplitudes, the HSG model reduces to the Ising model and the TBA system becomes trivial. With the help of the results on the free energy and the $g$-function for the Ising model [46, 47], one can derive the all-order expansion of the integral expression [12] of the remainder function:

$$
\begin{aligned}
R_{8} & =\frac{5 \pi}{4}-\frac{1}{2} \log \left(2 \cosh \frac{l \cos \varphi}{2}\right) \log \left(2 \cosh \frac{l \sin \varphi}{2}\right)+\frac{l^{2}}{8 \pi} \\
& +\pi \sum_{k=1}^{\infty}\left(\begin{array}{c}
\frac{1}{2} \\
k+1
\end{array}\right)\left(1-\frac{1}{2^{2 k+1}}\right) \zeta(2 k+1)\left(1-\frac{k+1}{2 k+1} f_{k}(\varphi)\right)\left(\frac{l}{\pi}\right)^{2 k+2},
\end{aligned}
$$


where $\zeta(z)$ is the zeta-function, $l, \varphi$ are given by the mass parameter as

$$
m=l e^{i \varphi},
$$

and the function $f_{k}(\varphi)$ are given through the hypergeometric function as

$$
f_{k}(\varphi):=\cos ^{2} \varphi_{2} F_{1}\left(-k, 1 ; \frac{1}{2}-k ; \sin ^{2} \varphi\right)+\sin ^{2} \varphi_{2} F_{1}\left(-k, 1 ; \frac{1}{2}-k ; \cos ^{2} \varphi\right) .
$$

We have refrained from expanding the logarithmic terms.

\subsection{Ten-point amplitudes}

For the ten-point amplitudes, there are two mass parameters $m_{1}, m_{2}$. In this case, the

consideration in the previous section completely determines the expansion up to and including terms of $\mathcal{O}\left(l^{4(1-\Delta)}\right)$ :

$$
R_{10}=R_{10}^{(0)}+R_{10}^{(4)} \cdot l^{8 / 5}+\mathcal{O}\left(l^{12 / 5}\right),
$$

where

$$
\begin{aligned}
& R_{10}^{(0)}=\frac{39}{20} \pi-\frac{5}{2} \log ^{2}\left(2 \cos \frac{\pi}{5}\right), \\
& R_{10}^{(4)}=\left(-\frac{1}{5} \tan \frac{\pi}{5}+C_{1}\right) \cdot\left|t_{1}^{(2,0)}\right|^{2},
\end{aligned}
$$

with

$$
\begin{aligned}
t_{1}^{(2,0)}= & C_{2}\left(\tilde{M}_{1}^{4 / 5}+\tilde{M}_{2}^{4 / 5}-C_{3} \tilde{M}_{1}^{2 / 5} \tilde{M}_{2}^{2 / 5}\right), \\
& C_{1}=20 \cos ^{4}\left(\frac{2 \pi}{5}\right)\left(1-5^{-1 / 2} \log \left(2 \cos \frac{\pi}{5}\right)\right), \\
& C_{2}=\frac{1}{4 \cdot 6^{1 / 5}} \Gamma(-1 / 5)\left[10 \cos \frac{\pi}{5} \gamma(3 / 5) \gamma(4 / 5)\right]^{1 / 2}, \\
& C_{3}=2-\left(\frac{3}{\pi^{2}}\right)^{1 / 5} \gamma(1 / 4)^{4 / 5},
\end{aligned}
$$

and $\gamma(x)=\Gamma(x) / \Gamma(1-x)$. This holds for the complex mass parameters,

$$
m_{s}=\tilde{M}_{s} l=e^{i \varphi_{s}}\left|\tilde{M}_{s}\right| l
$$

and $t_{1}^{(2,0)}$ is related to $\kappa_{n} G\left(\tilde{M}_{s}\right)$ by (4.17).

The results can be compared with the numerical results from the TBA equations (3.1). We show comparisons of the remainder function for various complex $m_{s}$ in Fig. 6 . We find a good agreement between our analytic expansions and the numerics. 


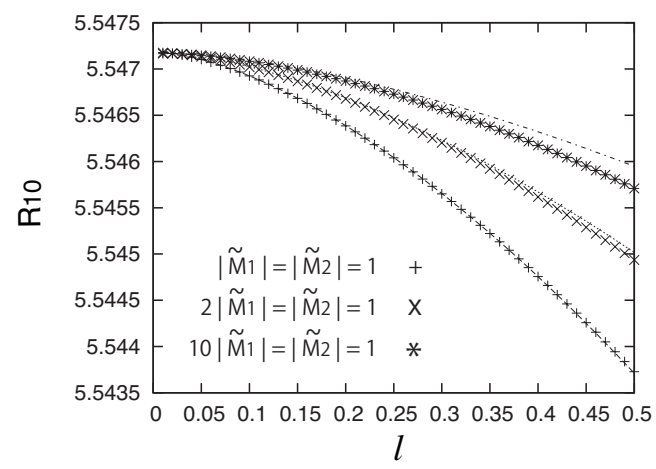

Figure 6: Comparisons of analytic results at strong coupling and numerics for various complex mass parameters with $\varphi_{1}=\pi / 20, \varphi_{2}=\pi / 5$. Dashed lines represent the analytic expansion (5.4)-(5.6), whereas the points $(+, \times, *)$ are from numerics.

\section{Comparison with two-loop results}

For the amplitudes corresponding to the minimal surfaces in $A d S_{3}$, the analytic expression of the remainder function has been given at two loops [31]. In this section, we compare this two-loop remainder function with that at strong coupling for the same cross-ratios [3, 4].

First, by expressing the cross-ratios by the Y-/T-functions, and then substituting their expansions into the two-loop result, one finds an expansion similar to (4.19):

$$
R_{2 n}^{2 \text {-loop }}=R_{2 n}^{2-\text { loop (0) }}+l^{\frac{8}{n}} R_{2 n}^{2-\text { loop (4) }}+\mathcal{O}\left(l^{\frac{12}{n}}\right) \text {. }
$$

The overall coupling constant $\lambda^{2}$ has been omitted above. For comparison, we next introduce a shifted and rescaled remainder function following Ref. [48,

$$
\bar{R}_{2 n}^{2 \text {-loop }}:=\frac{R_{2 n}^{2 \text {-loop }}-R_{2 n, \mathrm{UV}}^{2 \text {-loop }}}{R_{2 n, \mathrm{UV}}^{2 \text {-loop }}-(n-2) R_{6}^{2 \text {-loop }}},
$$

where $R_{2 n, \mathrm{UV}}^{2 \text {-loop }}$ are the remainder functions in the CFT/UV limit. This rescaled remainder function is calibrated so that it approaches 0 in the UV limit $(l \rightarrow 0)$ and -1 in the opposite IR limit $(l \rightarrow \infty)$. The rescaled remainder function at strong coupling, $\bar{R}_{2 n}^{\text {strong }}$, is defined similarly. It has been observed numerically that those remainder functions are close to each other for $2 n=8$ [48] .

Since the dependence on the mass parameters/momenta in the expansion up to $\mathcal{O}\left(l^{4(1-\Delta)}\right)$ is encoded in only one function, e.g., $t_{1}^{(2,0)} \propto \kappa_{n} G$, the ratio of the rescaled remainder functions at strong coupling and at two loops becomes just a number up to 
this order. For example, for lower-point amplitudes, we find that

$$
\begin{array}{ll}
\bar{\rho}_{8} \approx 1.0257, & \bar{\rho}_{10} \approx 0.9841, \\
\bar{\rho}_{12} \approx 0.9609, & \bar{\rho}_{14} \approx 0.9463, \\
\bar{\rho}_{16} \approx 0.9366, & \bar{\rho}_{18} \approx 0.9297,
\end{array}
$$

where $\bar{\rho}_{2 n}:=\bar{R}_{2 n}^{\text {strong }} / \bar{R}_{2 n}^{2 \text {-loop }}$. After some analysis, one can also find the asymptotic behavior,

$$
\bar{\rho}_{2 n} \rightarrow 0.905-\frac{0.118}{n} \quad(n \gg 1) .
$$

In principle, the ratio may take any values. We would thus like to say that the remainder functions at strong coupling and at two loops are (surprisingly) close for all $2 n$, though different.

\section{Summary}

In this talk, we discussed gluon scattering amplitudes/null-polygonal Wilson loops of four-dimensional $\mathcal{N}=4 \mathrm{SYM}$ at strong coupling. By the AdS/CFT correspondence, they are given by the minimal surfaces in $A d S_{5}$. Those minimal surfaces are in turn described by a set of integral equations. When the surfaces are contained in $A d S_{3}$ or $A d S_{4}$ subspace, those integral equations are identified with the TBA equations of the HSG model, which is an integrable deformation of the gauged WZW model by the weight 0 adjoint operators.

Based on the relation between the amplitudes and this underlying two-dimensional integrable model, we derived analytic expansions of the amplitudes/Wilson loops corresponding to the minimal surfaces in $A d S_{3}$ around the CFT/regular-polygonal limit. There were two non-trivial terms to be expanded in the remainder function, $A_{\text {free }}$ and $\Delta A_{\mathrm{BDS}}$. The former, $A_{\text {free}}$, is nothing but the free energy of the HSG model, and is expanded around the CFT limit by conformal perturbation. For the latter, $\Delta A_{\mathrm{BDS}}$, the sequential cross-ratios $c_{i, j}^{ \pm}$therein are directly expressed by the T-functions. This implies that every term in the remainder function nicely fits into the language of the integrable model. Interestingly, these T-functions are related to the $g$-function/boundary entropy, and the $g$-function, which is regarded as a boundary contribution to the free energy, is expanded around the CFT limit by conformal perturbation with boundary. Combining all the expansions together, we obtained the expansion of the remainder function. We also compared our analytic expansions at strong coupling with those at two loops. We found that the rescaled remainder functions at strong coupling and at two loops are close to each other for all $2 n$-point amplitudes. 


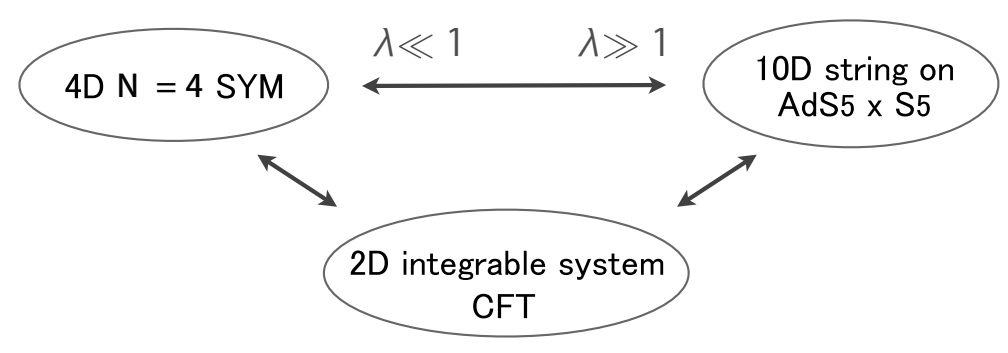

Figure 7: Two-, four- and ten-dimensional theories surrounding gluon scattering amplitudes.

For future, there still remain many questions to be addressed. For example, we still do not find a physical reason why the minimal surfaces are described by the TBA equations. The situation would be similar to the case of the ODE/IM correspondence [49. We do not find a physical reason for the relation between the $g$-and T-functions, either. It is also not clear why the rescaled remainder functions are so close to each other at strong coupling and at two loops. This seems to be suggesting some mechanism to constrain the amplitudes which is not yet understood. The understanding of such a mechanism, if any, would be important to uncover the full structure of the amplitudes to all orders.

Our analysis may be extended to the case of the amplitudes corresponding to the minimal surfaces in $A d S_{4}$. The integrable model for the general case of $A d S_{5}$, however, has not been identified. The precise relation between the perturbing operator and the mass parameters, i.e., the mass-coupling relation, which connects the conformal perturbation, the TBA system and the minimal surfaces/amplitudes, has not yet been obtained generally. These would be important questions for future. In this talk, we discussed the amplitudes in the strong-coupling limit. It would also be very interesting if one could incorporate the strong-coupling corrections and to interpolate the result to the weak-coupling side, as was the case for the spectral problem.

I would like to end this talk by showing one picture (Fig. 7). In this way, we see very interesting connections surrounding the gluon scattering amplitudes among the ten-dimensional string theory on $A d S_{5} \times S^{5}$, the four-dimensional SYM, and the twodimensional integrable systems (HSG model and Hitchin system) and CFTs. It would be interesting to explore these connections further. 


\section{Acknowledgments}

The author would like to thank the organizers for the opportunity to speak in this workshop. He would also like to thank Y. Hatsuda, K. Ito and K. Sakai for fruitful collaboration. His work is supported in part by Grant-in-Aid for Scientific Research from Ministry of Education, Culture, Sports, Science and Technology of Japan.

\section{References}

[1] Y. Hatsuda, K. Ito, K. Sakai and Y. Satoh, JHEP 1004 (2010) 108.

[2] Y. Hatsuda, K. Ito, K. Sakai and Y. Satoh, JHEP 1009 (2010) 064.

[3] Y. Hatsuda, K. Ito, K. Sakai and Y. Satoh, JHEP 1104 (2011) 100.

[4] Y. Hatsuda, K. Ito and Y. Satoh, JHEP 1202 (2012) 003.

[5] J. A. Minahan and K. Zarembo, JHEP 0303 (2003) 013.

[6] I. Bena, J. Polchinski and R. Roiban, Phys. Rev. D 69 (2004) 046002.

[7] N. Beisert, C. Ahn, L. F. Alday, Z. Bajnok, J. M. Drummond, L. Freyhult, N. Gromov and R. A. Janik et al., Lett. Math. Phys. 99 (2012) 3.

[8] Al. B. Zamolodchikov, Nucl. Phys. B 342 (1990) 695.

[9] Z. Bajnok, A. Hegedus, R. A. Janik and T. Lukowski, Nucl. Phys. B 827 (2010) 426.

[10] B. Eden, P. Heslop, G. P. Korchemsky, V. A. Smirnov and E. Sokatchev, Nucl. Phys. B 862 (2012) 123.

[11] L. F. Alday and J. M. Maldacena, JHEP 0706 (2007) 064.

[12] L. F. Alday and J. Maldacena, JHEP 0911 (2009) 082.

[13] L. F. Alday, D. Gaiotto and J. Maldacena, JHEP 1109 (2011) 032.

[14] L. F. Alday, J. Maldacena, A. Sever and P. Vieira, J. Phys. A 43 (2010) 485401.

[15] L. F. Alday and R. Roiban, Phys. Rept. 468 (2008) 153.

[16] J.M. Drummond, G.P. Korchemsky and E. Sokatchev, Nucl. Phys. B 795 (2008) 385.

[17] Z. Bern, L. J. Dixon and V. A. Smirnov, Phys. Rev. D 72 (2005) 085001.

[18] L. F. Alday and J. Maldacena, JHEP 0711 (2007) 068.

[19] J. M. Drummond, J. Henn, G. P. Korchemsky and E. Sokatchev, Nucl. Phys. B 826 (2010) 337.

[20] Z. Bern, L. J. Dixon, D. A. Kosower, R. Roiban, M. Spradlin, C. Vergu and A. Volovich, Phys. Rev. D 78 (2008) 045007. 
[21] J. M. Drummond, J. Henn, G. P. Korchemsky and E. Sokatchev, Nucl. Phys. B 815 (2009) 142.

[22] K. Sakai and Y. Satoh, JHEP 0910 (2009) 001.

[23] K. Sakai and Y. Satoh, JHEP 1003 (2010) 077.

[24] D. Gaiotto, G. W. Moore and A. Neitzke, Commun. Math. Phys. 299 (2010) 163.

[25] R. Koberle and J. A. Swieca, Phys. Lett. B 86 (1979) 209.

[26] C. R. Fernandez-Pousa, M. V. Gallas, T. J. Hollowood, J. L. Miramontes, Nucl. Phys. B 484 (1997) 609.

[27] Al. B. Zamolodchikov, Phys. Lett. B 253 (1991) 391.

[28] V. Del Duca, C. Duhr and V. A. Smirnov, JHEP 1005 (2010) 084.

[29] A. B. Goncharov, M. Spradlin, C. Vergu and A. Volovich, Phys. Rev. Lett. 105 (2010) 151605.

[30] V. Del Duca, C. Duhr and V. A. Smirnov, JHEP 1009 (2010) 015.

[31] P. Heslop and V. V. Khoze, JHEP 1011 (2010) 035.

[32] A. Kuniba, T. Nakanishi, J. Suzuki, J. Phys. A 44 (2011) 103001.

[33] V. V. Bazhanov, S. L. Lukyanov and A. B. Zamolodchikov, Commun. Math. Phys. 177 (1996) 381.

[34] P. Dorey, I. Runkel, R. Tateo and G. Watts, Nucl. Phys. B 578 (2000) 85.

[35] P. Dorey, A. Lishman, C. Rim and R. Tateo, Nucl. Phys. B 744 (2006) 239.

[36] I. Affleck, A. W. W. Ludwig, Phys. Rev. Lett. 67 (1991) 161.

[37] D. Gaiotto, J. Maldacena, A. Sever and P. Vieira, JHEP 1103 (2011) 092.

[38] F. Ravanini, R. Tateo, A. Valleriani, Int. J. Mod. Phys. A 8 (1993) 1707.

[39] C. R. Fernandez-Pousa and J. L. Miramontes, Nucl. Phys. B 518 (1998) 745.

[40] P. Dorey and J. L. Miramontes, Nucl. Phys. B 697 (2004) 405.

[41] Al. B. Zamolodchikov, Nucl. Phys. B 358 (1991) 497.

[42] H. Itoyama, P. Moxhay, Phys. Rev. Lett. 65 (1990) 2102.

[43] Al. B. Zamolodchikov, Int. J. Mod. Phys. A 10 (1995) 1125.

[44] Al. B. Zamolodchikov, Nucl. Phys. B 366 (1991) 122.

[45] V. A. Fateev, Phys. Lett. B 324 (1994) 45.

[46] T. R. Klassen and E. Melzer, Nucl. Phys. B 350 (1991) 635.

[47] P. Dorey, D. Fioravanti, C. Rim and R. Tateo, Nucl. Phys. B 696 (2004) 445.

[48] A. Brandhuber, P. Heslop, V. V. Khoze and G. Travaglini, JHEP 1001 (2010) 050 .

[49] P. Dorey, C. Dunning and R. Tateo, J. Phys. A 40 (2007) R205. 